\title{
The TENDL library: Hope, reality and future
}

\author{
D. Rochman ${ }^{1, a}$, A.J. Koning ${ }^{2,3}$, J.Ch. Sublet ${ }^{4}$, M. Fleming ${ }^{4}$, E. Bauge ${ }^{5}$, S. Hilaire ${ }^{5}$, P. Romain ${ }^{5}$, B. Morillon ${ }^{5}$, H. Duarte ${ }^{5}$, \\ S. Goriely ${ }^{6}$, S.C. van der Marck ${ }^{7}$, H. Sjöstrand ${ }^{3}$, S. Pomp ${ }^{3}$, N. Dzysiuk ${ }^{7}$, O. Cabellos ${ }^{8}$, H. Ferroukhi ${ }^{1}$, and A. Vasiliev ${ }^{1}$ \\ 1 Reactor Physics and Systems Behaviour Laboratory, Paul Scherrer Institut, Switzerland \\ 2 Nuclear Data Section, International Atomic Energy Agency, Vienna, Austria \\ 3 Uppsala University, Uppsala, Sweden \\ ${ }^{4}$ United Kingdom Atomic Energy Authority, Abingdon, UK \\ 5 CEA DAM DIF, Service de Physique Nucléaire, France \\ ${ }^{6}$ Université Libre de Bruxelles, 1050 Brussels, Belgium \\ 7 NRG, Petten, The Netherlands \\ ${ }^{8}$ OECD Nuclear Energy Agency, Paris, France
}

\begin{abstract}
The TALYS Evaluated Nuclear Data Library (TENDL) has now 8 releases since 2008 . Considerable experience has been acquired for the production of such general-purpose nuclear data library based on the feedback from users, evaluators and processing experts. The backbone of this achievement is simple and robust: completeness, quality and reproducibility. If TENDL is extensively used in many fields of applications, it is necessary to understand its strong points and remaining weaknesses. Alternatively, the essential knowledge is not the TENDL library itself, but rather the necessary method and tools, making the library a side product and focusing the efforts on the evaluation knowledge. The future of such approach will be discussed with the hope of nearby greater success.
\end{abstract}

\section{Introduction}

The TALYS Evaluated Nuclear Data Library project (TENDL) started in 2008. Since then, a new version was released every year, including further improvements and increasing its relevance for diverse applications. Such project includes nuclear data evaluations for neutron, proton, deuteron, triton, helium-3, alpha, and photon induced reactions. To illustrate the evolution, the TENDL2008 library included 350 isotopic evaluations from ${ }^{19} \mathrm{~F}$ to ${ }^{209}$ Po for neutron induced reactions, whereas the TENDL2015 library includes more than 2800 evaluations for neutron (all isotopes from ${ }^{1} \mathrm{H}$ to ${ }^{289} \mathrm{Fl}$ leaving longer than $1 \mathrm{sec}$.) up to $200 \mathrm{MeV}$ with covariance files, fission yields, thermal scattering data and so-called random files for uncertainty propagation. Other outputs, such as processed files ready to be used in simulation codes are also available. An overview of the TENDL evolution in terms of file number is presented in Fig. 1. The process of producing such library is presented in Ref. [1].

Over the years, TENDL is being used in many subjects, among which fission, fusion, medical isotope production, astrophysics, high-energy physics, damage calculations, non proliferation, etc. For more references on the use of TENDL in different application fields, see the latest TENDL webpage. As specific examples, one can cite the inclusion of TENDL in the projected JEFF-3.3 library, in the GEANT simulation program, as well as in the CASMO library.

One of the difference with other existing nuclear data libraries is that TENDL itself is "only" the output of series

a e-mail: dimitri-alexandre.rochman@psi.ch of codes, referred to as T6. T6 stands for six codes, such as TALYS, TEFAL, TASMAN, TARES, TAFIS and TANES, with specific tasks for each one in order to produce an ENDF-6 file as complete as possible. Therefore the development of TENDL goes through the improvements of T6, assuring reproducibility. Once such a system is in place, the developers, the evaluators, theoreticians and other specialists can focus on the essentials: improving the methods, theories, models and parameters. With this approach, the improvement of the quality is almost guaranteed, with better agreement to observables, better physics and better tracability.

The T6 approach also helped to produce other interesting methods, such as the Total Monte Carlo idea (TMC) [2], fast TMC [3] and recently the Bayesian Monte Carlo (BMC) $[4,5]$. These methods are now in use for both the development of TENDL, but also in the uncertainty propagation in reactor physics related subjects. Other projects, aiming at a global improvement of the nuclear data and their applications were also proposed, such as in Ref. [6].

Amid such recent successes, the possibilities for improvements are growing. One reason is that as TENDL is more used, more parts of the evaluations are tested by different people, using different simulation tools and additional feedback indicates potential areas of improvements. Another reason is that T6 is not yet a production tool as such. It is constantly being developed, therefore mixing production and development. In the future, a prioritization of the improvements needs to be achieved following the users' demand and not specifically the developers's wishes, together with a better separation between developments and production. 


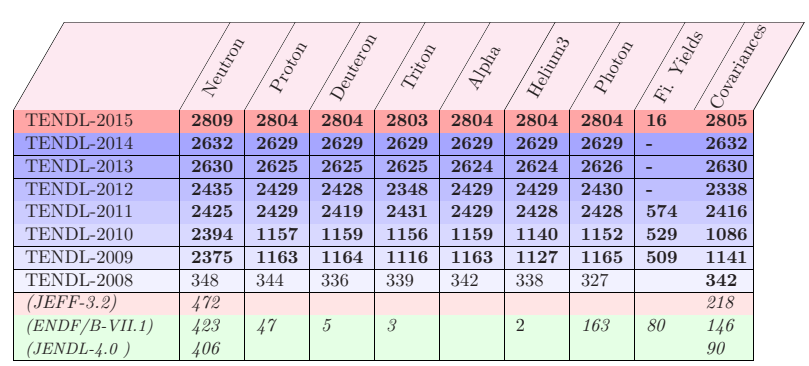

Figure 1. Numbers of ENDF-6 files as a function of the TENDL versions.

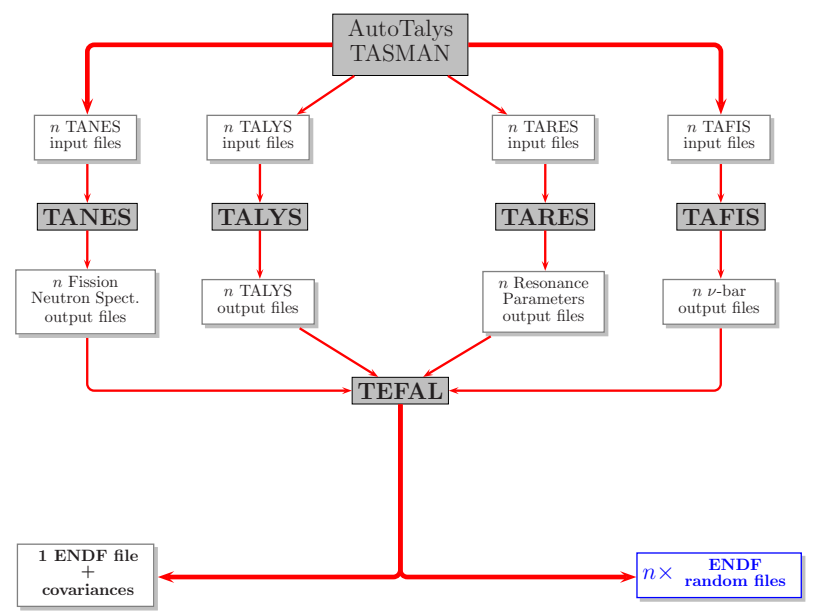

Figure 2. The T6 tool and what it produces: one or more ENDF-6 files.

The different points shortly presented in this introduction will be detailed in the following. For more details on the different subjects, the readers are invited to visit the presented references.

\section{T6}

As mentioned, T6 is made of a suite of programs, each of them with specific tasks. T6 was originally created as TALYS does not produce (yet) all the necessary quantities which need to be calculated for a complete ENDF-6 file. Ultimately, T6 should be come T5, T4 until T1 as all parts will be integrated into a single software. Currently, efforts are being made to merge TALYS, TASMAN and TEFAL into a single code. If successful, the result of this unification will change T6 in T4. This would allow an easier maintenance and more practical use. But for the time being, the T6 package is mainly composed of six codes, plus some other utility packages. A simplified structure is presented in Fig. 2. TENDL can be produced every year with improved performances because of the robustness and constant improvements of the T6 package. Many of the T6 programs are already widely known, and details can be found in Ref. [1]. Recently, some noticeable improvements were made, for instance in the generation of covariances with updated parameter distributions based on experimental data (the BMC method, see next section), and the retroactive method with the use of the Reich Moore limited in the resonance range (starting after the 2015 release). Also, every single isotope in TENDL since 2013 has a unique set of resonances, for both ground states and

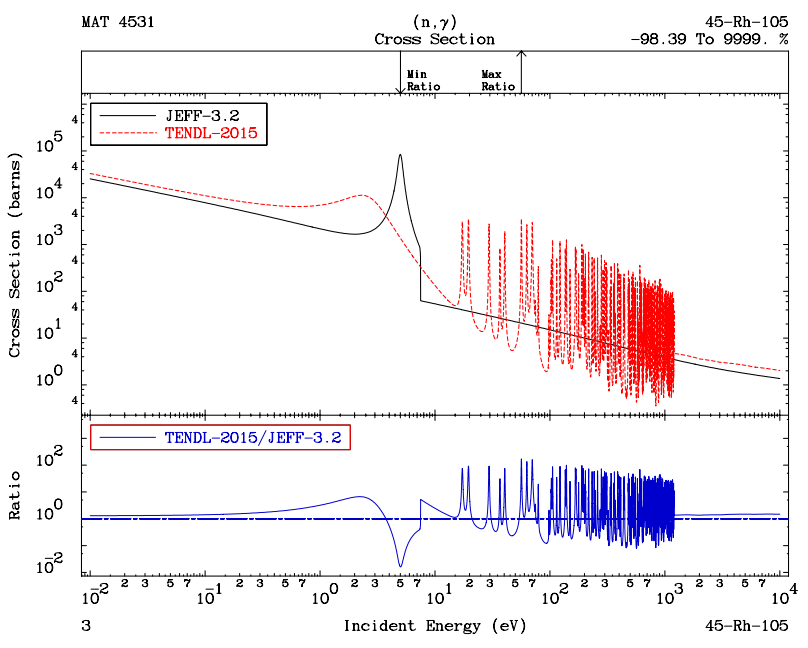

Figure 3. Non-representative example of the good quality of the TENDL-2015 evaluations for ${ }^{105} \mathrm{Rh}$.

possible isomers, following the "High Fidelity Resonance" method as presented in Ref. [7,8].

As a (biased) example of the good performance of TENDL-2015 and the latest resonance parameters included in $\mathrm{T} 6$, the comparison for ${ }^{105} \mathrm{Rh}$ between TENDL-2015 and JEFF-3.2 is presented in Fig. 3. This example is likely to be not representative of the quality of JEFF-3.2.

Another important feature of the TENDL libraries is that the same format is used for all evaluations, which allows similar processing for all isotopes. As well, the inclusion for all isotopes of the so-called MF6, including all double differential data, together with the information on all emitted particles for activation purposes is bringing some unique features that no other libraries have. More details on these subjects can be found in the given references and will not be repeated here. But the importance of T6 in the TENDL methodology is crucial: it represents the sum of the knowledge leading to TENDL.

\section{Related projects: TMC, BMC, HFR}

Different methods step in during the elaboration of the TENDL library. As presented many times, the random variation of all model parameters leads to random cross sections and other quantities, which can also be formatted into ENDF-6 files. This allows to loop over simulation code using nuclear data, thus applying the TMC method. As an improvement on TMC to include differential information, the BMC method allows to sample from a updated parameter distributions, based on simple weighting functions. Finally the use of statistical resonances for all isotopes (the HFR method) brings the TENDL evaluations for short-lived isotopes to "the next level". Recent progress in these three methods, strongly linked to TENDL, and again possible thanks to the robustness of T6, are shortly presented in the following.

\subsection{TMC}

The TMC method has been presented and applied many times. Variations exist depending on different levels of simplifications [9-12] and using different background [13]. The TMC method, based on any version 


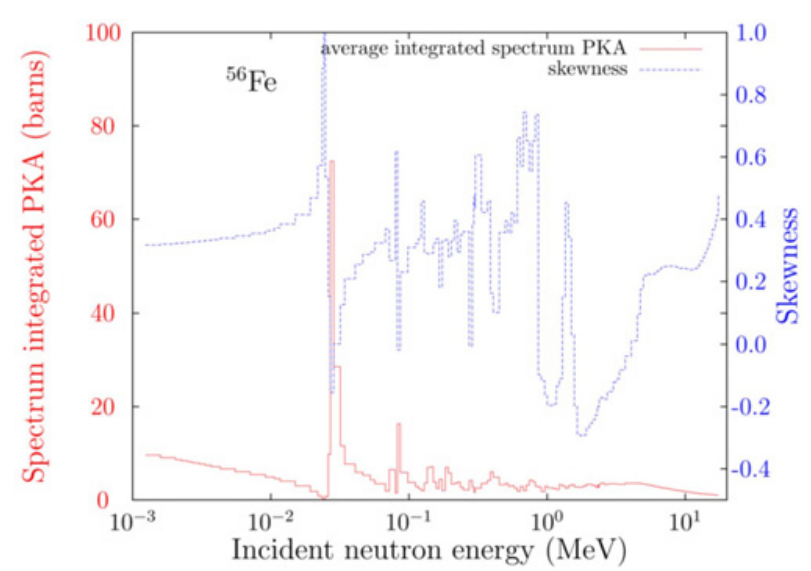

Figure 4. Example of the TMC for damage calculations: PKA and its skewness for ${ }^{56} \mathrm{Fe}$.

of TENDL, allows to propagate nuclear data uncertainties from the basic knowledge (the Schrödinger equation) to large-scale facilities (e.g. reactor transients or fuel storage for a million year). The different versions of the simplified TMC, often using cross section covariance files, allow to reach the same goal, but starting from probabilities of interactions (cross sections) instead of wave functions of the given quantum system.

The TMC method, contrary to Generalized Least Squared methods (GLS), gives access to a probability density function for the calculated observables, with more than the usual two moments (average and standard deviation) provided by the GLS method. Other quantities, if defined, such as the skewness can be calculated, with potential impacts on safety assessments. If quantities such as the average or standard deviations are not defined (e.g. as for Cauchy or Breit-Wigner distributions), then the median and median absolute deviation quantities, more statistically robust, can be used instead. Many examples of the application of TMC can be found in the literature; as one example, Fig. 4 presents the skewness of the spectrum integrated Primary Knock-on Atom (PKA) for ${ }^{56} \mathrm{Fe}$, illustrating a case of varying skewness as a function of the neutron incident energy. The uncertainties calculated in the TMC method are directly related to the TENDL library and the method used to generate random ENDF-6 files. Until 2014, the method to create such files was a simple sampling from uniform or Normal distributions for model parameters, with or without accept/reject method (see [1] for details). It was nevertheless noticed in Ref. [14] that such approach does not use the experimental correlations (as sometimes presented in EXFOR) and that TMC is not Bayesian. Although the goal of TMC was not to follow a Bayesian method, such deficiencies are addressed in the BMC approach since 2015.

\section{2. $B M C$}

The origin of the Bayesian Monte Carlo method, or BMC, was presented in the so-called BFMC method [15], developed in UMC-B [14] and generalized in Ref. [4]. The connection between the different methods is presented in e.g. Ref. [16], chapter 4. The idea is relatively simple to understand and follows the logic of sampling model parameters as well as experimental data, as presented in Refs. [17,18]. In the iteration number 0 ,

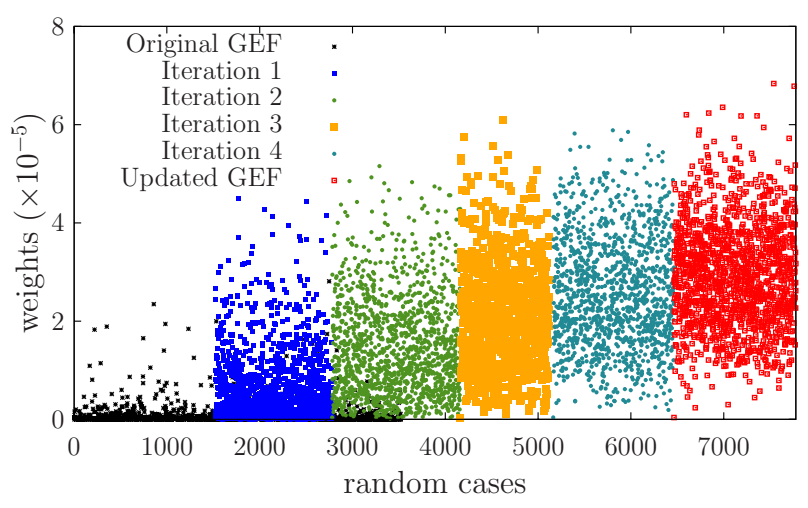

Figure 5. Examples of distributions of weights taken from Ref. [5].

all model parameters are sampled uniformly with large standard deviations, large enough to cover all experimental data. After sampling, each calculated cross section is compared to the experimental data and weights $\omega$ are calculated based on the distance between the two values $\left(\omega \propto \exp \left(-\chi^{2} / 2\right)\right)$. These weights are then reported to the sampled parameters to update their probability distributions (pdf). This way, in the new iteration number 1 , the model parameters are sampled from updated arbitrary pdf, as defined with the previous weights. This new sampling will lead to a new set of calculated cross sections, which are closer to the experimental data than the ones from the iteration number 0 . This way, the updated parameter distributions in iteration 1 take into account the theoretical and experimental information.

A continuation of this process is proposed in Ref. [5], where the process is repeated $n$ times, leading to iteration $2, \ldots, n$. An example of the convergence of the process in terms of weights is presented in Fig. 5. By using a large number of iterations, the parameter pdf will converge either due to the perfect agreement with experimental data (within their uncertainties), which is relatively unlikely given the model defects or the experimental errors, or due to the theoretical limit to agree with the experimental data.

For the TENDL library, the parameter update is performed with the iteration number 1 . The calculated covariances and the random files, as presented in Fig. 2 reflect therefore both the theoretical and experimental information. This feature, applied to all isotopes with differential data, is unique to the TENDL library.

\subsection{HFR}

For the majority of important isotopes for reactor simulations, the amount of experimental information is often adequate. In the resonance range, the parameters of the measured and evaluated resonances (usually from a fit to the available data) are used to describe the resonance behavior of the cross sections. In the case where no experimental data are available (for instance because the half-life of the isotope is too short, or because its natural occurrence is small), the usual approach is to extend the Hauser-Feshbach calculation to the low energy region and thus to obtain smooth cross sections. In the previous activation libraries (such as the EAF libraries), a different approach was used with the single resonance approximation (SRA): a fake and 


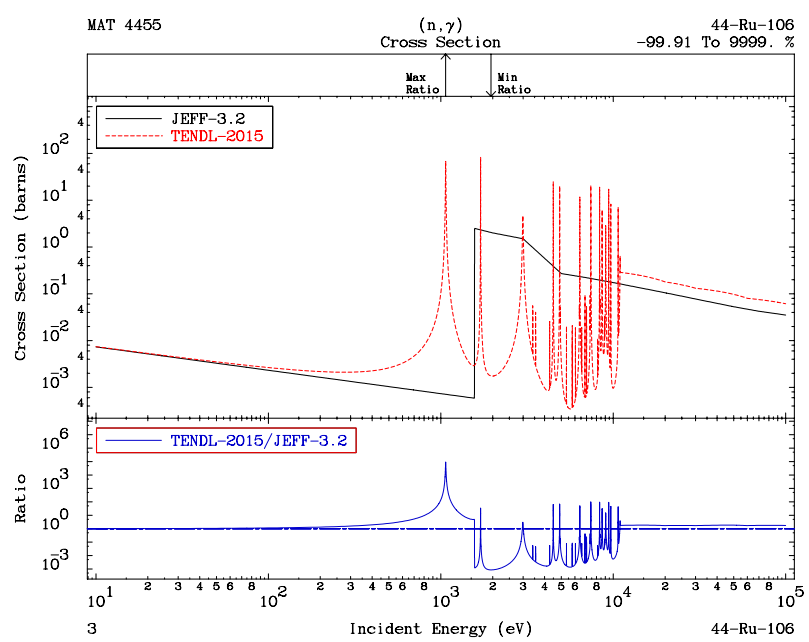

Figure 6. Example of reconstructed statistical resolved resonances for ${ }^{106} \mathrm{Ru}$ from TENDL-2015, compared to JEFF-3.2.

unique resonance was created in order to reproduce some existing integral information (such as the thermal cross section or the resonance integral). Recently an extension of the SRA approach is used in TENDL, as described in Ref. [8]. The average information from the HauserFeshbach calculations (average widths, energy spacing between resonances for each orbital angular momentum and spin of resonance state) is used to generate a set of statistical resonances. Such method allows to have a consistency between the resonance and the fast neutron range, as the statistical resonances are derived from the same calculation parameters as in the fast neutron range. An example is provided in Fig. 6 for the capture cross section of ${ }^{106} \mathrm{Ru}$. This feature has some important implications mainly outside the usual reactor applications, where short-lived isotopes can have an important impact. The HFR is also applied to isomers when included in TENDL (isomers living longer than $1 \mathrm{sec}$ ). Alternatively, the HFR was tested over 3300 isotopes (from the stability line to the neutron drip line), showing expected behaviors for neutron-rich isotopes important in astrophysics [19].

\section{What's next?}

The TENDL libraries have been a successful experience, together with the development of the necessary tools and methods. Still, many improvements can be realized. Considering that the goal of any nuclear data library is to solve the following equation

$$
\chi^{2}=0 \text { or } 1
$$

where $\chi^{2}$ is a simple measure of deviation between experiments and calculations, the TENDL library and its method can offer a practical solution. The choice of $\chi^{2}=0$ or 1 depends on the target community. For pure application, the best adjustment is obtained when the calculations overlap with the experimental data. In this case, " 0 " is the target. From the point of view of nuclear data evaluation, the solution of $\chi^{2}=1$ is satisfactory, and avoids any overprediction (or over-fitting) of the experimental data. Such possibilities were detailed in Ref. [20] with specific applications for ${ }^{239} \mathrm{Pu}$ and the
$\mathrm{Cu}$ isotopes in Refs. [21,22]. The idea is again relatively simple once the T6 programs are in place. First a set of experimental data needs to be selected. It should be both differential and integral data, including as much sources as possible. The starting point library can be any library, such as TENDL, JEFF, ENDF/B or JENDL. The most sensitive isotopes are likely to be ${ }^{235} \mathrm{U}$ and ${ }^{238} \mathrm{U}$, therefore these isotopes (at the same time) needs to be optimized. To do this, random evaluations for these isotopes can be produced with T6. The other ones included in the starting point library stay unique. Each combination of the random ${ }^{235} \mathrm{U}$ and ${ }^{238} \mathrm{U}$ evaluations with the library is then used to be compared with the set of experimental data and $\chi^{2}$ values are calculated for each random library. The combination with the minimum $\chi^{2}$ is selected as the best library. The next step is to repeat the same process, this time keeping ${ }^{235} \mathrm{U}$ and ${ }^{238} \mathrm{U}$ equal to the selected ones, and using random evaluations for the second most sensitive isotopes (for instance ${ }^{239} \mathrm{Pu}$ ). New random libraries are then produced, leading to new $\chi^{2}$ values. Again, the one with the minimum $\chi^{2}$ is selected. This process is repeated for all sensitive isotopes. After such loop on all isotopes, a new one can be performed until convergence of the whole process. This approach, although computer intensive, is not out of reach with large computer clusters and can lead to a real minimization of $\chi^{2}$, almost numerically solving Eq. (1).

For the time being, improvements are still needed at the level of T6. With the increasing number of tests on the TENDL files from many different users, some hidden deficiencies regularly appear. They often concern specific parts of the ENDF-6 file which can be interpreted differently by processing codes. This ambiguous interpretation of the ENDF-6 rules can only be solved with the developers of such processing codes.

A more obvious source of limitation in the TENDL library is the lack of parameter adjustments for some specific reactions. This leads for instance to cross sections with poor agreement to experimental data and thus degrades the quality of the evaluation. Such disagreement can also come from the limitation of the theoretical models used in T6. Theoretical models always include some degrees of simplifications, which is ultimately limiting the quality of TENDL. Other libraries can bypass this issue by manually correct the evaluations at a final stage. In the case of TENDL, this action is not permitted and other solutions need to be found. For the time being, a process called "autonorm" allows to apply normalization factors in TALYS at the level of transmission coefficients in order to reproduce "any" cross section. One of the short-term goal for the coming TENDL versions is to limit the number of these autonorm calls and to replace them as much as possible by a better physical model with better adjusted parameters.

Related to the other library projects, TENDL is making use of the other evaluations, e.g. with the autonorm process. It is recognized that the current quality of TENDL would be different without the interactions with the other libraries. For instance, the major actinides are being imported from ENDF/B-VII.1 or JEFF-3.2 [23,24]. For many activation and dosimetry reactions, the IRDFF library is an important reference source [25]. Also, the interaction with the fusion community made a number of advances possible [26]. To be complete, one needs 
to acknowledge that the current state of knowledge for resonance parameters as well as for model parameters in the fast neutron range is reliable in TENDL (and other libraries) thanks to various past efforts, such as experimental compilations [27,28], and the RIPL model parameter database [29].

Finally, a more general limitation for TENDL and for some other nuclear data libraries is the lack of coordinated effort and funding. Both limit the number of specialists working on a given problem and lead to ill-defined priorities. Such an issue can only be solved once the importance of nuclear data is recognized by the industry and safety bodies.

\section{Conclusion}

The TENDL libraries and their associated methods have hopefully modified the nuclear data landscape, leading to general improvements regarding both fundamental and applied simulations. Enhanced simulations at the level of a nuclear data library are still possible and ideas exist to reach $\chi^{2}=0$. As local improvements of nuclear data files are not enough, the union of forces are required to achieve such goal, more than ever.

\section{References}

[1] A. Koning, D. Rochman, Nuclear Data Sheets 113(12), 2841 (2012)

[2] A. Koning, D. Rochman, Ann. Nucl. Energy 35, 2024 (2008)

[3] D. Rochman, W. Zwermann, S. van der Marck, A. Koning, H. Sjostrand, P. Helgesson, B. KrzykaczHausmann, Nucl. Sci. Eng. 177, 337 (2014)

[4] A. Koning, Eur. Phys. J. A 51, 184 (2015)

[5] D. Rochman, O. Leray, A. Vasiliev, H. Ferroukhi, A. Koning, M. Fleming, J. Sublet, Ann. Nucl. Energy 95, 125 (2016)

[6] E. Bauge, M. Dupuis, S. Hilaire, S. Péru, A. Koning, D. Rochman, S. Goriely, Nucl. Data Sheets 118, 32 (2014)

[7] P. Ribon, Annals of Nuclear Energy 13(4), 209 (1986)

[8] D. Rochman, A. Koning, J. Kopecky, J.C. Sublet, P. Ribon, M. Moxon, Annals of Nuclear Energy 51, 60 (2013)

[9] W. Zwermann, L. Gallner, M. Klein, B. KrzykaczHausmann, I. Pasichnyk, A.P. nd K. Velkov, Eur. Phys. J. Web of Conf. 42, 03003 (2013)

[10] O. Buss, A. Hoefer, J. Neuber, NUDUNA Nuclear Data Uncertainty Analysis, in Proceedings of the Intl. Conf. on Nuclear Criticality (ICNC 2011) (2011) edinburgh, Scotland, September. 19-22, on CD-ROM
[11] N. Garcia-Herranz, O. Cabellos, F. Alvarez-Velarde, J. Sanz, E. González-Romero, J. Juan, Ann. Nucl. Energy 37, 1570 (2010)

[12] T. Zhu, A. Vasiliev, H. Ferroukhi, A. Pautz, Ann. Nucl. Energy 109, 713 (2015)

[13] R. Capote, D. Smith, A. Trkov, M. Meghzifene, Journal of ASTM International 9, 104115 (2012)

[14] R. Capote, D.L. Smith, A. Trkov, EPJ Web of Conferences 8, 04001 (2010)

[15] E. Bauge, S. Hilaire, P. Dossantos-Uzarralde, Evaluation of the covariance matrix of neutronic cross sections with the Backward-Forward Monte Carlo method, in Proceedings of the International Conference on Nuclear Data for Science and Technology (2007) Nice, France, April 22-27

[16] G. Schnabel, Ph.D. thesis, Technical University, Vienna, Austria (2015)

[17] P. Helgesson, H. Sjostrand, A. Koning, D. Rochman, E. Alhassan, S. Pomp, Nucl. Data Sheets 123, 214 (2015)

[18] D. Rochman, A. Koning, E. Bauge, A. Plompen, Annals of Nuclear Energy 73, 7 (2014)

[19] D. Rochman, S. Goriely, A. Koning, H. Ferroukhi, Physics Letters B 764, 109 (2017)

[20] D. Rochman, A.J. Koning, S.C. van der Marck, Eur. Phys. J. A 51, 1 (2015)

[21] D. Rochman, A. Koning, Nucl. Sci. Eng. 169, 68 (2011)

[22] D. Rochman, A. Koning, Nucl. Sci. Eng. 170, 265 (2012)

[23] M.B. Chadwick, M. Herman, P. Obložinský, M.E. Dunn, Y. Danon, A.C. Kahler, D.L. Smith, B. Pritychenko, G. Arbanas, R. Arcilla et al., Nucl. Data Sheets 112, 2887 (2011)

[24] A.J. Koning, E. Bauge, C.J. Dean, E. Dupont, U. Fischer, R.A. Forrest, R. Jacqmin, H. Leeb, M.A. Kellett, R.W. Mills et al., Journal of the Korean Physical Society 59(2), 1057 (2011)

[25] R. Capote, K. Zolotarev, V. Pronyaev, A. Trkov, Journal of ASTM International 9, 104119 (2012)

[26] U. Fischer, M. Avrigeanu, V. Avrigeanu, O. Cabellos, I. Kodeli, A. Koning, A. Konobeyev, H. Leeb, D. Rochman, P. Pereslavtsev et al., Nuclear Data Sheets 120, 226 (2014)

[27] S. Mughaghab, Atlas of Neutron Resonances (Elsevier, 2006)

[28] N. Otuka, E. Dupont, V. Semkova, B. Pritychenko, A. Blokhin, M. Aikawa, S. Babykina, M. Bossant, G. Chen, S. Dunaeva et al., Nuclear Data Sheets 120, 272 (2014)

[29] R. Capote, M. Herman, P. Obložinský, P. Young, S. Goriely, T. Belgya, A. Ignatyuk, A. Koning, S. Hilaire, V. Plujko et al., Nuclear Data Sheets 110(12), 3107 (2009) 